\title{
CENTRAL VENOUS PRESSURE AND HEART RATE PREDICT MICROVASCULAR FUNCTION AND DISEASE SEVERITY IN CHILDREN WITH MENINGOCOCCAL SEPTIC SHOCK
}

\author{
M. Festa ${ }^{1}$, J. Gamble ${ }^{2}$, S. Nadel ${ }^{3}$
}

${ }^{1}$ Paediatric Intensive Care, The Children's Hospital at Westmead, Sydney, NSW, Australia, ${ }^{2}$ Department of Physiology, Imperial College, ${ }^{3}$ Paediatric Intensive Care, St Mary's Hospital at Imperial College, London, $U K$

Background and aims: This research describes microvascular function in infants and children with meningococcal septic shock (MSS). The microvascular parameter, isovolumetric venous pressure (Pvi), the equilibrium point for oedema formation, provides information on the dynamic state of the microcirculation. Pvi is correlated with disease severity scores and haemodynamic parameters routinely available in the paediatric intensive care unit.

Methods: Pvi was measured using venous congestion plethysmography (VCP) in the leg of 28 intubated and ventilated infants and children on day 1 of MSS. Measured parameters are related to Glasgow Meningococcal Septicaemia Prognostic Score (GMSPS) and Sequential Organ Failure Assessment (SOFA) score, and to routine measures of haemodynamic status obtained from conventional cardiovascular monitoring.

Results: Median age 5.5 years (IQR 2.6 - 9.9; range 0.4 - 15.6). Median GMSPS 11 (4 - 15) and day 1 SOFA score $8\left(\right.$ IQR 6 - 10). Pvi correlates with disease severity $\left(r^{2}=0.22\right)$ and organ dysfunction scores $\left(\mathrm{r}^{2}=\right.$ 0.24). A strong positive correlation is observed between heart rate (HR) and Pvi $\left(r^{2}=0.31\right)$, perfusion pressure and Pvi $\left(r^{2}=0.35\right)$, and central venous pressure $(C V P)$ and Pvi $\left(r^{2}=0.26\right)$. Bivariate linear regression demonstrates the combination of HR and CVP is highly predictive of Pvi $\left(\left(r^{2}=0.48\right)\right.$.

Conclusion: This study is the first to demonstrate a relationship between Pvi and organ dysfunction and disease severity in children with MSS. Pvi provides important information on the state of the microcirculation in septic shock, and may be predicted by routinely monitored haemodynamic variables. 